\title{
NISTIR 7163
}

Experimentally Measured Total X-Ray Attenuation Coefficients Extracted from Previously Unprocessed Documents Held by the NIST Photon and Charged Particle Data Center. II.

Hubbell, J. H.

U. S. DEPARTMENT OF COMMERCE Technology Adininistration National Institute of Standards and Technology

Gaithersburg, MD 20899-8463 


\section{Experimentally Measured Total X-Ray Attenuation Coefficients Extracted from Previously Unprocessed Documents Held by the NIST Photon and Charged Particle Data Center. II.}

Hubbell, J. H.

U. S. DEPARTMENT OF COMMERCE Technology Administration National Institute of Standards and Technology

Gaithersburg, MD 20899-8463

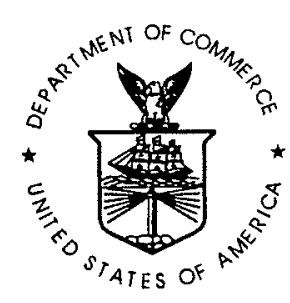

U.S. DEPARTMENT OF COMMERCE Donald L. Evans, Secretary TECHNOLOGY ADMINISTRATION Phillip J. Bond, Under Secretary for Technology NATIONAL INSTITUTE OF STANDARDS AND TECHNOLOGY

Arden L. Bement, Jr., Director 


\section{Introduction:}

Since 1950 the National Institute of Standards and Technology (NIST; formerly National Bureau of Standards) has maintained a data base of measured and theoretical x-ray attenuation and cross section data in a hard-copy file of original source documents in the form of reprints, reports, and personal communications. This data base extends over 11 decades of photon energies, from $10 \mathrm{eV}$ (extreme ultraviolet or XUV region) to $100 \mathrm{GeV}$ (cosmic-ray energy region). The purpose of this data base, now residing in the NIST Photon and Charged Particle Data Center (PCPDC), is to provide photon (XUV, x-ray, gamma-ray, and bremsstrahlung) interaction data required in a variety of medical, industrial, defense, and basic-science applications. This NIST/PCPDC data base, unique in the global scientific community, has been used from time to time as the basis for the tabulation of photon cross sections and attenuation coefficients [1-15] and related quantities such as the photon energytransfer and energy-absorption coefficients [5-7, 9, 11, 16-18].

In collaboration with $\mathrm{H}$. Gerstenberg, E. Saloman and a series of summer student assistants, the data extracted from the accumulated NIST/PCPDC-held documents (1907-1986) were keyboarded into machine-readable form in 1986 [19-24]. In a further report, NISTIR 5893 (1996) [25], to which this report is a sequel, the data from additional documents (1981-1995) were extracted and put in machine-readable and standardized form. The present report is a further supplement and update to the data base, incorporating data from further previously unprocessed documents (1978-2004) accumulated in the PCPDC collection and listed and annotated (photon energy ranges, elements measured, and uncertainty estimates) in Section $\mathrm{V}$.

\section{Procedures:}

In the above hard-copy file of original source documents, the measurements were made in a great variety of contexts and scientific disciplines, for different purposes. These purposes range from medical $\mathrm{x}$-ray therapy and diagnostic applications to $\mathrm{x}$-ray crystallography and aircraft radiometric fuel gauge development, and include many other extremely diverse human endeavors requiring, in common, reliable values of this $\mathrm{x}$-ray interaction data. Hence the measured results in these source documents were given by the authors in units peculiar to the particular scientific discipline in which the measurements were taken.

For example, in much of the x-ray crystallography and $\mathrm{x}$-ray fluorescence (XRF) analytical literature, and also in much of the atomic physics basic research literature, the photon energies are not given, but rather the photon wavelengths, in units of angstroms $(\AA)$ or nanometers $(\mathrm{nm})$. The photon interaction probabilities (cross sections) are also given in a variety of different forms, such as linear attenuation coefficients (e.g., $\left.\mathrm{cm}^{-1}\right)$, mass attenuation (or absorption) coefficients (e.g., $\mathrm{cm}^{2} / \mathrm{g}$ or $\mathrm{m}^{2} / \mathrm{kg}$ ), cross sections (e.g., barns per atom [b/atom], or $\mathrm{Mb}$ per atom, or $\mathrm{Mb}$ per molecule for binary gases, etc.). In previous 
photon energies $9.6 \mathrm{MeV}$ to $162 \mathrm{MeV}$, in addition to many-point measurements also for $\mathrm{Cu}$, $\mathrm{Sn}$ and $\mathrm{Ta}$ in that same source reference [78Gi01]. In this current report, the extreme case for density and number of data points for a single element are the 502 cross section measurements in $\mathrm{W}(\mathrm{Z}=74)$ from $1.453 \mathrm{keV}$ to $2.353 \mathrm{keV}$ by Levine et al. [003Le01], followed closely by the 501 measurements in $\mathrm{Si}(\mathrm{Z}=14)$ from $1.800 \mathrm{keV}$ to $2.300 \mathrm{keV}$ by Owens et al. [0020w02].

Table 1 is summarized in Table 2, showing for each element the number of papers which contained data for that element, and the total number of data points extracted from these papers. From the 23 papers examined, for all elements, a grand total of 3357 data points were extracted.

Authors' estimates of uncertainty, if provided, are noted in the square brackets at the end of each annotated reference in the document listing in Section V. These uncertainties vary greatly, from a lower bound of $\pm 0.1 \%$ in Roy et al. [97Ro01] to an upper bound of $\pm 300 \%$ in Tikkanen and Huovelin [96Ti01]. In general, a more meaningful "envelope of uncertainty" is obtained by noting differences between independent measurements for the same element and energies under different experimental conditions.

\section{Text References:}

1. White (Grodstein), G.R., X-Ray Attenuation Coefficients from $10 \mathrm{kev}$ to $100 \mathrm{MeV}$, NBS Rep. 1003 (1952).

2. Fano, U., Gamma-Ray Attenuation, Part 1, Nucleonics 11 (8), 8-12 (1953); Part 2, 11 (9), 55-60 (1953).

3. Grodstein, G.R., X-ray Attenuation Coefficients from $10 \mathrm{kev}$ to $100 \mathrm{MeV}$, NBS Circ. 583 (1957).

4. McGinnies, R.T., X-Ray Attenuation Coefficients from $10 \mathrm{keV}$ to $100 \mathrm{MeV}$, Suppl. to NBS Circ. 583 (1959).

5. Berger, R.T. (McGinnies), The X- or Gamma-Ray Energy Absorption or Transfer Coefficient: Tabulations and Discussion, Radiat. Res. 15, 1-29 (1961).

6. Hubbell, J.H. and Berger, M.J., Sec. 4.1: Attenuation Coefficients, Energy Absorption Coefficients, and Related Quantities (p. 167-184) and Sec. 4.2: Photon Atomic Cross Sections (p. 185-202). IAEA Engineering Compendium on Radiation Shielding, R.G. Jaeger, ed., Springer, Berlin (1968).

7. Hubbell, J.H., Photon Cross Sections, Attenuation Coefficients, and Energy 
Coefficients, Rad. Res. 136, 147-170 (1993).

18. Seltzer, S.M. and Hubbell, J.H., Tables and Graphs of Photon Mass Attenuation Coefficients and Mass Energy Absorption Coefficients for Photon Energies $1 \mathrm{keV}$ to $20 \mathrm{MeV}$ for Elements $\mathrm{Z}=1$ to 92 and Some Dosimetric Materials, Japanese Society of Radiological Technology Report ISSN 1340-7716 (1995); see also Hubbell. J.H. and Seltzer, S.M., National Institute of Standards and Technology Report NISTIR 5632 (1995).

19. Gerstenberg, H.M., and Hubbell, J.H., Comparison of Experimental with Theoretical Photon Attenuation Cross Sections Between $10 \mathrm{eV}$ and $100 \mathrm{GeV}$ (p. 1007-1009), Nuclear Data for Science and Technology, K.H. Bockhoff, ed., ECSC, EEC, EAEC, Brussels and Luxembourg (1983).

20. Saloman, E.B., and Hubbell, J.H., X-Ray Attenuation Coefficients (Total Cross Sections): Comparison of the Experimental Data Base with the Recommended Values of Henke and the Theoretical Values of Scofield for Energies Between 0.1-100 keV, National Bureau of Standards Internal Report NBSIR 86-3431 (1986).

21. Hubbell, J.H., Gerstenberg, H.M. and Saloman, E.B., Bibliography of Photon Total Cross Section (Attenuation Coefficient) Measurements $10 \mathrm{eV}$ to $13.5 \mathrm{GeV}$, National Bureau of Standards Internal Report NBSIR 86-3461 (1986).

22. Saloman, E.B., and Hubbell, J.H., Critical Analysis of Soft X-Ray Cross Section Data, (Presented at the Third International Symposium on Radiation Physics, Ferrara, Italy, Sept. 30-Oct. 4, 1985), Nucl. Instr. Meth. A $\underline{255}$, 38-42 (1987).

23. Saloman, E.B., Hubbell, J.H. and Scofield, J.H., X-Ray Attenuation Cross Sections for Energies $100 \mathrm{eV}$ to $100 \mathrm{keV}$ and Elements $\mathrm{Z}=1$ to $\mathrm{Z}=92$, Atomic Data and Nuclear Data Tables $\underline{38}, 1-197$ (1988).

24. Saloman, E.B., Hubbell, J.H. and Berger, M.J., National Bureau of Standards Data Base of Photon Absorption Cross Sections from $10 \mathrm{eV}$ to $100 \mathrm{GeV}$, Proc. SPIE 911 , 100-106 (1988).

25. Hubbell, J.H., Experimentally Measured Total X-Ray Attenuation Coefficients Extracted from Previously Unprocessed Documents Held by the NIST Photon and Charged Particle Data Center (I.), NISTIR 5893 (1996).

26. Hubbell, J.H., Bibliography of Photon Total Cross Section (Attenuation Coefficient) Measurements $10 \mathrm{eV}$ to $13.5 \mathrm{GeV}, 1907-1993$, NISTIR 5437 (1994). 
001An01 Angelone, M., Bubba, T. and Esposito, A., Appl. Radiat. Isot. 55, 505-511 (2001) Measurement of the Mass Attenuation Coefficient for Elemental Materials in the Range $6 \leq \mathrm{Z} \leq 82$ Using X-Rays from 13 up to $50 \mathrm{keV}$

(13.37 kev to $50.65 \mathrm{keV}$ : C, Al, Ti, V, Mn, Fe, Co, Ni, Cu, Zn, Zr, Nb, Mo, Rh, $\mathrm{Pd}, \mathrm{Ag}, \mathrm{Cd}, \mathrm{In}, \mathrm{Ta}, \mathrm{Pt}, \mathrm{Au}, \mathrm{Pb})[ \pm 2.5 \%$ to $8.0 \%]$

001Ba01 Baltazar-Rodrigues, J. and Cusatis, C., Nucl. Instr. Meth. B 179, 325-333 (2001) Determination of X-Ray Photoelectric Absorption of Ge and Si Avoiding Solid-State Effects $(8.048 \mathrm{keV}$ to $24.945 \mathrm{keV}: \mathrm{Si}, \mathrm{Ge})[ \pm 0.1 \%$ to $0.3 \%$ ]

001Ch01 Chantler, C.T., Tran, C.Q., Barnea, Z., Paterson, D., Cookson, D.J. and Balaic, D.X., Phys. Rev. A 64, 062506, 1-15 (2001)

Measurement of the X-Ray Mass Attenuation Coefficient of Copper Using 8.85-20 $\mathrm{keV}$ Synchrotron Radiation

(8.8709 $\mathrm{keV}$ to $20.0268 \mathrm{keV}: \mathrm{Cu})$ [0.271\% to $0.332 \%$ ]

002Ma01 Mallikarjuna, M.L., Gowda, S.B.A., Krishnaveni, S., Gowda, R. and Umesh, T.K., Nucl. Sci. \& Eng. 140, 96-102 (2002)

Studies on Photon Interaction around the K-Edge of Some Elements (5.895 $\mathrm{keV}$ to $84.3 \mathrm{keV}: \mathrm{Cu}, \mathrm{Zr}, \mathrm{Ag}, \mathrm{Sn})$ [ $\pm 3 \%$ to $4 \%$ ]

002Ma02 Mallikarjuna, M/L., Gowda, S.B.A., Gowda, R. and Umesh, T.K., Radiat. Phys. Chem. 65, 217-223 (2002)

Studies on Photon Interaction around the K-Edge of some Rare-Earth Elements (6.4 keV to $84.3 \mathrm{keV}$ : O, La, Ce, Pr, Nd, Sm, Gd, Dy, Ho, Er) [ $\pm 3 \%$ to $4 \%$ ]

002Ow01 Owens, A., Fraser, G.W. and Gurman, S.J., Radiat. Phys. Chem. 65, 109-121 (2002)

Near K-Edge Linear Attenuation Coefficients for $\mathrm{Si}, \mathrm{SiO}_{2}$ and $\mathrm{Si}_{3} \mathrm{~N}_{4}$ Note: Measurement results are presented for both crystalline ("Si-c") and amorphous ("Si-a"). For presentation in this report the crystalline-sample "Si-c" results were selected.

$(1.800 \mathrm{keV}$ to $2.300 \mathrm{keV}: \mathrm{Si})$ [ $\pm \%$ not given]

002Su01 Suzuki, I.H. and Saito, N., J. Electron Spectrosc. 123, 239-245 (2002)

Photoabsorption Cross Section of $\mathrm{Kr}$ in the Sub-keV Energy region $(100 \mathrm{eV}$ to $1300 \mathrm{eV}: \mathrm{Kr})[ \pm 1 \%]$

002 Ta01 Tamura, M., Akimoto, T., Aoki, Y., Ikeda, J., Sato, K., Fujita, F., Homma, A., Sawamura, T. and Narita, M., Nucl. Instr. Meth. A 484, 642-649 (2002)

Measurement of Mass Attenuation Coefficients around the $\mathrm{K}$ absorption Edge by Parametric X-Rays

(17.22 keV to $20.56 \mathrm{keV}: \mathrm{Nb}, \mathrm{Zr}, \mathrm{Mo})$ [ $\pm 1 \%$ to $15 \%$ ] 
Table 1. Experimental Cross Section Data Extracted from Documents.

$\mathrm{Z}=5, \mathrm{~B}$

$5.110+051.415+00$

$1.330+066.203-01$

$\mathrm{Z}=6, \mathrm{C}$

$2.834+024.168+04$

$2.842+023.212+05$

$2.850+021.075+06$

$2.858+024.205+05$

$2.866+023.413+05$

$2.880+026.307+05$

$2.900+027.750+05$

$2.920+021.187+06$

$2.940+021.416+06$

$2.960+021.579+06$

$2.980+021.404+06$

$3.000+021.434+06$

$3.200+027.726+05$

$3.400+026.467+05$

$3.600+025.811+05$

$3.800+025.299+05$

$4.000+02 \quad 4.774+05$

$\mathrm{Z}=6, \mathrm{C}$

$8.041+039.973+01$

$\mathrm{Z}=6, \mathrm{C}$

$1.337+042.200+01$

$3.206+045.000+00$

$\mathrm{Z}=7, \mathrm{~N}$

$4.020+023.328+05$

$4.040+028.120+05$

$4.060+021.402+06$

$4.080+021.738+06$

$4.100+021.331+06$

$4.120+021.333+06$

$4.140+021.087+06$

$4.160+027.987+05$
$\mathrm{eV}$, barns per atom pairs

5 pts $\quad$ ref $=003 \mathrm{Te} 01$

$6.620+051.303+00 \quad 1.170+069.864-01 \quad 1.280+067.460-01$

$\mathrm{eV}$, barns per atom pairs

$65 \mathrm{pts} \quad$ ref $=96 \mathrm{Ti} 01$

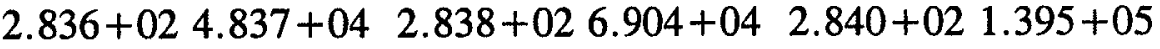

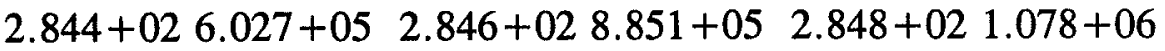

$2.852+028.326+05 \quad 2.854+026.128+05 \quad 2.856+024.578+05$

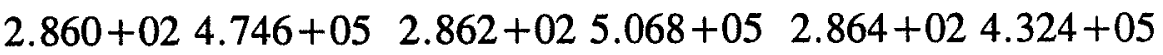

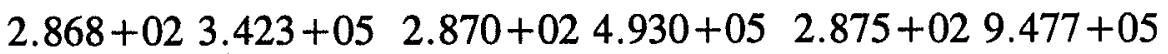

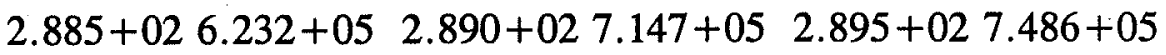

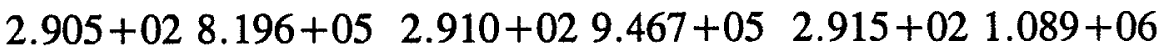

$\begin{array}{llllll}2.925+02 & 1.227+06 & 2.930+02 & 1.267+06 & 2.935+02 & 1.341+06\end{array}$

$\begin{array}{llllll}2.945+02 & 1.482+06 & 2.950+02 & 1.545+06 & 2.955+02 & 1.585+06\end{array}$

$\begin{array}{llllll}2.965+02 & 1.535+06 & 2.970+02 & 1.478+06 & 2.975+02 & 1.430+06\end{array}$

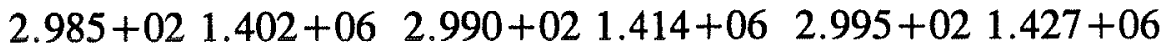

$3.050+021.387+06 \quad 3.100+029.697+05 \quad 3.150+028.225+05$

$3.250+027.608+05 \quad 3.300+027.314+05 \quad 3.350+026.828+05$

$3.450+026.177+05 \quad 3.500+025.978+05 \quad 3.550+025.866+05$

$3.650+025.746+05 \quad 3.700+025.629+05 \quad 3.750+025.463+05$

$3.850+025.153+05 \quad 3.900+025.008+05 \quad 3.950+024.860+05$

$\mathrm{eV}$, barns per atom pairs

$1 \mathrm{pt} \quad \mathrm{ref}=004 \mathrm{Ec} 01$

$\mathrm{eV}$, barns per atom pairs

$8 \mathrm{pts} \quad$ ref $=001 \mathrm{An} 01$

$1.744+04 \quad 1.150+01 \quad 2.210+047.200+00 \quad 2.499+046.100+00$

$3.665+044.200+00$

$4.423+044.030+005.065+043.900+00$

$\mathrm{eV}$, barns per atom pairs

77 pts $\quad$ ref $=96 \mathrm{Ti0} 1$

$4.025+023.117+05 \quad 4.030+024.431+05 \quad 4.035+027.422+05$

$4.045+027.656+05$

$4.050+028.918+05$

$4.055+021.146+06$

$4.065+021.597+06$

$4.070+021.674+06$

$4.075+021.681+06$

$4.085+021.783+06$

$4.090+021.688+06$

$4.095+021.495+06$

$4.105+021.251+06$

$4.110+021.261+06$

$4.115+021.302+06$

$4.125+021.316+06$

$4.130+021.264+06$

$4.135+021.186+06$

$4.145+029.882+05$

$4.150+029.030+05$

$4.155+028.399+05$

$4.165+027.835+05$

$4.170+027.685+05$

$4.175+027.663+05$ 
$\mathrm{Z}=8, \mathrm{O}$

$6.400+036.036+02$

$2.414+041.504+01$

$6.430+044.890+00$

$8.430+044,380+00$
$\mathrm{eV}$, barns per atom pairs

$8.041+033.303+02$

$3.080+049.630+00$

$1.053+041.355+02$

$3.500+048.010+00$

$7.287+044.630+00$

$7.083+044.670+00$

$8.100+044.430+00$
$\mathrm{Z}=10, \mathrm{Ne}$

$4.490+017.730+06$

$6.990+016.120+06$

$9.500+014.230+06$

$1.150+023.140+06$

$1.350+022.330+06$

$1.550+021.780+06$

$1.750+021.350+06$

$2.100+029.030+05$

$2.500+025.840+05$

$2.900+024.010+05$

$3.300+022.870+05$

$3.700+022.180+05$

$4.200+021.570+05$

$5.000+029.800+04$

$5.800+026.600+04$

$6.500+024.950+04$

$7.200+023.650+04$

$7.800+023.040+04$

$8.479+022: 330+04$

$8.800+023.550+05$

$8.950+023.300+05$

$9.050+023.310+05$

$9.200+023.220+05$

$9.600+022.890+05$

$1.100+032.020+05$

$1.265+031.360+05$

$$
\mathrm{eV} \text {, barns per atom pairs }
$$

103 pts $\quad$ ref $=003 \mathrm{Su} 01$

$4.990+017.920+06 \quad 5.490+017.530+06 \quad 5.990+016.810+06$

$7.980+015.200+068.600+014.910+06$

$1.000+023.940+061.050+023.650+06$

$1.200+022.920+06$

$1.400+022.180+06$

$1.250+022.710+06$

$9.000+014.560+96$

$1.100+023.380+06$

$1.300+022.510+06$

$1.600+021.660+06$

$1.450+022.040+06$

$1.500+021.910+06$

$1.800+021.270+06$

$1.650+02 \quad 1.550+06$

$1.700+021.450+06$

$2.200+028.160+05$

$2.600+025.300+05$

$1.900+021.130+06$

$2.000+021.000+06$

$2.300+027.310+05$

$2.700+024.840+05$

$2.400+026.500+05$

$3.000+023.700+05$

$3.400+022.670+05$

$3.100+023.400+05$

$2.800+024.420+05$

$3.800+022.040+05$

$3.500+022.490+05$

$3.200+023.120+05$

$4.400+021.390+05$

$3.900+021.910+05$

$3.600+022.340+05$

$5.200+028.870+04$

$4.600+02 \quad 1.230+05$

$4.000+021.790+05$

$5.400+028.010+04$

$4.800+021.100+05$

$6.000+026.070+04$

$6.200+025.610+04$

$5.600+027.250+04$

$6.600+024.740+04$

$6.800+024.340+04$

$6.400+025.160+04$

$7.400+023.410+04$

$7.500+023.310+04$

$7.000+023.980+04$

$8.000+022.850+04$

$8.200+022.670+04$

$7.600+023.230+04$

$8.588+022.280+04$

$8.700+023.760+05$

$8.400+022.560+04$

$8.850+023.450+05$

$8.900+023.370+05$

$8.750+023.670+05$

$8.975+023.290+05$

$9.000+023.290+05$

$8.925+023.330+05$

$9.075+023.310+05$

$9.100+023.290+05$

$9.025+023.300+05$

$9.300+023.130+05$

$9.400+023.050+05$

$9.150+023.260+05$

$9.800+022.710+05$

$1.000+032.560+05$

$9.500+022.970+05$

$1.150+031.820+05$

$1.200+031.600+05$

$1.050+032.260+05$

$1.290+031.270+05$

$1.316+031.260+05$

$1.240+031.460+05$

$\mathrm{Z}=13, \mathrm{Al}$

$\mathrm{eV}$, barns per atom pairs

$291 \mathrm{pts} \quad$ ref $=96 \mathrm{Ti0} 1$

$6.000+011.022+06$

$6.400+016.550+05$

$6.800+015.420+05$

$7.150+015.610+05$

$7.190+015.612+05$

$7.230+015.689+05$

$7.270+011.287+06$

$7.310+011.878+06$

$6.100+015.173+05$

$6.500+016.345+05$

$6.200+015.719+05$

$6.900+015.309+05$

$7.160+015.608+05$

$6.600+015.223+05$

$7.000+015.358+05$

$6.300+016.193+05$

$7.170+015.598+05$

$6.700+015.229+05$

$7.200+015.614+05$

$7.210+015.630+05$

$7.100+015.585+05$

$7.240+015.758+05$

$7.250+015.923+05$

$7.180+015.617+05$

$7.280+011.763+06$

$7.290+011.720+06$

$7.220+015.653+05$

$7.320+012.136+06$

$7.330+012.125+06$

$7.260+016.828+05$

$7.350+012.061+06$

$7.360+012.042+06$

$7.370+012.031+06$

$7.300+011.682+06$

$7.340+012.087+06$

$7.380+012.029+06$ 
$\mathrm{Z}=13, \mathrm{Al}$ $\mathrm{eV}$, barns per atom pairs

$31 \mathrm{pts} \quad$ ref $=004 \mathrm{Le} 01$

$4.000+031.631+04 \quad 4.200+03 \quad 1.417+04 \quad 4.400+03 \quad 1.243+04 \quad 4.600+031.106+04$ $4.800+039.810+03$ $5.600+036.358+03$ $6.400+034.276+03$ $7.200+033.030+03$ $8.000+032.204+03$ $8.800+031.677+03$ $9.500+031.299+03$ $5.000+038.728+03 \quad 5.200+037.728+03$ $5.800+035.646+036.000+035.148+03$ $6.600+033.924+03 \quad 6.800+033.596+03$ $5.400+036.879+03$ $7.000+033.341+03$ $\begin{array}{llllll}7.400+03 & 2.710+03 & 7.600+03 & 2.583+03 & 7.800+03 & 3.347+03\end{array}$ $\begin{array}{llllll}8.200+03 & 2.017+03 & 8.400+03 & 1.935+03 & 8.600+03 & 1.807+03\end{array}$ $\begin{array}{llllll}9.000+03 & 1.582+03 & 9.100+03 & 1.491+03 & 9.300+03 & 1.391+03\end{array}$ $9.700+031.231+03 \quad 9.900+031.166+03$

$\mathrm{Z}=13, \mathrm{Al}$ $6.400+034.310+03$
$\mathrm{eV}$, barns per atom pairs $8.041+032.263+03$
2 pts $\quad$ ref $=97 \mathrm{Ke} 01$
$\mathrm{Z}=13, \mathrm{Al}$

$1.337+044.400+02$ $2.210+041.120+02$ $4.423+042.070+01$
$\mathrm{eV}$, barns per atom pairs $1.497+043.180+02$ $2.499+047.800+01$ $5.065+041.630+01$ (1)
$\mathrm{Z}=14, \mathrm{Si}$

$1.800+031.414+04$ $1.804+031.397+04$ $1.808+031.381+04$ $1.812+031.376+04$
$\mathrm{eV}$, barns per atom pairs

$1.744+042.300+02 \quad 1.963+041.540+02$ $3.206+044.100+013.665+043.000+01$

$\begin{array}{lllllllll}2.810+02 & 1.330+06 & 2.812+02 & 1.328+06 & 2.814+02 & 1.328+06 & 2.816+02 & 1.326+06 \\ 2.818+02 & 1.325+06 & 2.820+02 & 1.327+06 & 2.822+02 & 1.327+06 & 2.824+02 & 1.328+06 \\ 2.826+02 & 1.330+06 & 2.828+02 & 1.333+06 & 2.830+02 & 1.338+06 & 2.832+02 & 1.350+06 \\ 1.554+03 & 1.905+04 & 1.555+03 & 2.424+04 & 1.556+03 & 3.662+04 & 1.557+03 & 6.007+04 \\ 1.558+03 & 8.841+04 & 1.559+03 & 1.243+05 & 1.560+03 & 1.604+05 & 1.561+03 & 1.904+05 \\ 1.562+03 & 2.110+05 & 1.563+03 & 2.246+05 & 1.564+03 & 2.339+05 & 1.565+03 & 2.383+05 \\ 1.566+03 & 2.367+05 & 1.567+03 & 2.341+05 & 1.568+03 & 2.333+05 & 1.569+03 & 2.312+05 \\ 1.570+03 & 2.320+05 & 1.571+03 & 2.300+05 & 1.572+03 & 2.261+05 & 1.573+03 & 2.202+05 \\ 1.574+03 & 2.107+05 & 1.575+03 & 2.046+05 & 1.576+03 & 1.980+05 & 1.577+03 & 1.933+05 \\ 1.578+03 & 1.829+05 & 1.579+03 & 1.780+05 & 1.580+03 & 1.707+05 & 1.581+03 & 1.657+05 \\ 1.582+03 & 1.600+05 & 1.583+03 & 1.549+05 & 1.584+03 & 1.512+05 & 1.585+03 & 1.467+05 \\ 1.586+03 & 1.449+05 & 1.587+03 & 1.470+05 & 1.588+03 & 1.527+05 & 1.589+03 & 1.558+05 \\ 1.590+03 & 1.662+05 & 1.591+03 & 1.739+05 & 1.592+03 & 1.803+05 & 1.593+03 & 1.840+05 \\ 1.594+03 & 1.866+05 & 1.595+03 & 1.889+05 & 1.596+03 & 1.876+05 & 1.597+03 & 1.852+05 \\ 1.598+03 & 1.843+05 & 1.599+03 & 1.827+05 & 1.600+03 & 1.803+05 & 1.610+03 & 1.701+05 \\ 1.620+03 & 1.474+05 & 1.630+03 & 1.609+05 & 1.640+03 & 1.636+05 & 1.650+03 & 1.608+05 \\ 1.660+03 & 1.482+05 & 1.670+03 & 1.479+05 & 1.680+03 & 1.494+05 & 1.690+03 & 1.509+05 \\ 1.700+03 & 1.504+05 & 1.710+03 & 1.436+05 & 1.720+03 & 1.391+05 & 1.730+03 & 1.367+05 \\ 1.740+03 & 1.349+05 & 1.750+03 & 1.347+05 & 1.760+03 & 1.316+05 & 1.770+03 & 1.284+05 \\ 1.780+03 & 1.267+05 & 1.790+03 & 1.248+05 & 1.800+03 & 1.232+05 & & \end{array}$
$2.818+021.325+06$
$2.812+021.328+06 \quad 2.814+02 \quad 1.328+06$
$2.824+021.328+06$
$2.828+021.333+06$
$.830+021.338+06$ 
$1.992+031.196+05 \quad 1.993+03 \quad 1.195+05$ $1.996+03 \quad 1.191+05 \quad 1.997+03 \quad 1.190+05$ $2.000+031.186+052.001+031.185+05$ $2.004+031.184+05 \quad 2.005+031.184+05$ $2.008+03 \quad 1.185+05 \quad 2.009+03 \quad 1.187+05$ $2.012+031.193+052.013+031.194+05$ $2.016+031.196+05 \quad 2.017+031.196+05$ $2.020+031.197+05 \quad 2.021+031.197+05$ $2.024+031.197+05 \quad 2.025+031.197+05$ $2.028+031.197+05 \quad 2.029+031.194+05$ $2.032+031.184+05 \quad 2.033+031.181+05$ $2.036+031.184+05$ $2.040+031.184+05$ $2.044+031.177+05$ $2.048+031.163+05$ $2.052+031.150+05$ $2.056+031.144+05$ $2.060+031.142+05$ $2.064+031.138+05$ $2.068+031.129+05$ $2.072+031.120+05$ $2.076+031.111+05$ $2.080+031.103+05$ $2.084+031.096+05$ $2.088+031.092+05$ $2.092+031.089+05$ $2.096+031.080+05$

$2.100+031.081+05$ $2.104+031.078+05$ $2.108+031.079+05$

$2.112+031.079+05$

$2.116+031.079+05$

$2.120+031.078+05$

$2.124+031.076+05$

$2.128+031.075+05$

$2.132+031.069+05$

$2.136+031.059+05$

$2.140+031.050+05$

$2.144+031.042+05$

$2.148+031.036+05$

$2.152+031.031+05$

$2.156+031.027+05$

$2.160+031.022+05$

$2.164+031.018+05$
$1.994+031.194+05$

$1.998+031.188+05$

$2.002+031.184+05$

$2.006+031.184+05$

$2.010+031.184+05$

$2.014+031.195+05$

$2.018+031.197+05$

$2.022+031.197+05$

$2.026+031.197+05$

$2.030+031.190+05$

$2.034+031.182+05$

$2.038+031.187+05$

$2.042+031.182+05$

$2.046+031.170+05$

$2.050+031.157+05$

$2.054+031.146+05$

$2.058+031.142+05$

$2.062+031.141+05$

$2.066+031.133+05$

$2.070+031.124+05$

$2.074+031.115+05$

$2.078+031.107+05$

$2.082+031.099+05$

$2.086+031.094+05$

$2.090+031.091+05$

$2.094+031.088+05$

$2.098+031.089+05$

$2.102+031.079+05$

$2.106+031.079+05$

$2.110+031.079+05$

$2.114+031.079+05$

$2.118+031.078+05$

$2.122+031.077+05$

$2.126+031.075+05$

$2.130+031.072+05$

$2.134+031.064+05$

$2.138+031.054+05$

$2.142+031.046+05$

$2.146+031.039+05$

$2.150+031.134+05$

$2.154+031.028+05$

$2.158+031.025+05$

$2.162+031.020+05$

$2.166+031.016+05$
$1.995+031.193+05$

$1.999+031.187+05$ $2.003+031.183+05$ $2.007+031.184+05$ $2.011+031.191+05$ $2.015+031.195+05$ $2.019+031.197+05$ $2.023+031.198+05$ $2.027+031.197+05$ $2.031+031.187+05$ $2.035+031.183+05$ $2.039+031.185+05$ $2.043+031.181+05$ $2.047+031.166+05$ $2.051+03.1 .153+05$ $2.055+031.145+05$ $2.059+031.142+05$ $2.063+031.141+05$ $2.067+031.131+05$ $2.071+031.122+05$ $2.075+031.113+05$ $2.079+031.105+05$ $2.083+031.097+05$ $2.087+031.093+05$ $2.091+031.090+05$ $2.095+031.087+05$ $2.099+031.083+05$ $2.103+031.078+05$ $2.107+031.079+05$ $2.111+031.079+05$ $2.115+031.079+05$ $2.119+031.078+05$ $2.123+031.077+05$ $2.127+031.075+05$ $2.131+031.070+05$ $2.135+031.062+05$ $2.139+031.052+05$ $2.143+031.044+05$ $2.147+031.037+05$ $2.151+031.032+05$ $2.155+031.027+05$ $2.159+031.024+05$ $2.163+031.019+05$ $2.167+031.015+05$ 
$8.113+032.872+03$ $8.514+032.488+03$

$8.915+032.174+03$

$9.316+03 \quad 1.916+03$

$9.716+031.692+03$

$1.002+041.542+03$

$1.032+041.413+03$

$1.072+041.267+03$

$1.112+041.134+03$

$1.152+041.024+03$

$1.192+049.245+02$

$1.232+048.359+02$

$1.292+047.239+02$

$1.352+046.324+02$

$1.412+045.589+02$

$1.472+044.917+02$

$1.523+044.455+02$

$1.603+043.831+02$

$1.663+043.446+02$

$1.743+042.987+02$

$1.803+042.688+02$

$1.863+042.439+02$

$1.942+042.159+02$

$\mathrm{Z}=14, \mathrm{Si}$

$8.048+032.922+03$

$2.216+041.467+02$

$\mathrm{Z}=16, \mathrm{~S}$

$6.400+039.263+03$

$\mathrm{Z}=20, \mathrm{Ca}$

$6.400+032.103+04$

$\mathrm{Z}=20, \mathrm{Ca}$

$5.110+055.831+00$

$1.330+063.650+00$

$\mathrm{Z}=22, \mathrm{Ti}$

$\mathrm{eV}$, barns per atom pairs

6 pts ref $=001 \mathrm{Ba} 01$

$8.906+032.172+03$

$2.495+041.045+04$

$1.744+041.900+03$

$\mathrm{Z}=23, \mathrm{~V}$

$6.400+033.715+04 \quad 8.041+032.152+04$
$\mathrm{eV}$, barns per atom pairs

$\mathrm{eV}$, barns per atom pairs

$8.041+031.121+04$

$\mathrm{eV}$, barns per atom pairs

$8.041+035.042+03$

$2.210+049.000+02$

$3.206+043.310+02$

$4 \mathrm{pts}$ ref $=001 \mathrm{An} 01$

$\mathrm{eV}$, barns per atom pairs

2 pts ref $=97 \mathrm{Ke} 01$

2 pts ref $=97 \mathrm{Ke} 01$

5 pts $\quad$ ref $=003 \mathrm{Te} 01$

$6.620+055.242+00 \quad 1.170+063.909+00 \quad 1.280+063.758+00$

$4.423+041.320+02$

2 pts ref $=97 \mathrm{Ke} 01$ 
$\mathrm{Z}=29, \mathrm{Cu}$

$5.895+031.262+04$ $1.440+048.480+03$

$5.201+042.560+02$ $8.100+047.700+01$

$\mathrm{Z}=29, \mathrm{Cu}$

$6.400+031.015+04$

$\mathrm{Z}=29, \mathrm{Cu}$

$8.871+034.009+03$

$9.013+033.157+04$

$9.053+033.050+04$

$9.093+033.071+04$

$9.133+033.225+04$

$9.333+032.857+04$

$9,835+032.427+04$

$1.064+041.964+04$

$1.145+041.612+04$

$1.245+041.287+04$

$1.326+041.086+04$

$1.407+049.277+03$

$1.487+047.977+03$

$1.548+047.171+03$

$1.568+046.934+03$

$1.648+046.053+03$

$1.729+045.317+03$

$1.770+044.995+03$

$1.850+044.423+03$

$1.891+044.167+03$

$1.968+043.733+03$
$\mathrm{eV}$, barns per atom pairs

$\begin{array}{llllll}6.400+03 & 1.007+04 & 8.041+03 & 5.389+03 & 1.053+04 & 1.989+04\end{array}$

$2.414+042.077+03$

$3.080+041.070+03$

$3.500+047.550+02$

$6.430+041.440+02$

$8.430+046.900+01$

$7.083+041.110+027.287+041.020+02$
$\mathrm{eV}$, barns per atom pairs

$8.041+035.487+03$

$\mathrm{eV}$, barns per atom pairs

$8.972+034.154+03$

$9.023+032.962+04$

$8.982+031.844+04$

$9.033+033.329+04$

$9.073+033.254+04$

$9.113+033.009+04$

$9.103+032.930+04$

$9.183+033.064+04$

$9.233+032.994+04$

$9.434+032.759+04$

$9.384+032.823+04$

$9.836+032.437+04$

$1.084+041.866+04$

$1.004+042.297+04$

$1.104+041.775+04$

$1.165+041.540+04$

$1.185+041.470+04$

$1.266+041.233+04$

$1.346+041.044+04$

$1.427+048.931+03$

$1.507+047.705+03$

$1.286+041.180+04$

$1.366+041.002+04$

$1.447+048.592+03$

$1.527+047.424+03$

$1.558+047.045+03$

$1.568+046.931+03$

$1.588+046.691+03$

$1.608+046.475+03$

$1.669+045.848+03$

$1.750+044.876+03$

$1.689+045.670+03$

$1.770+045.001+03$

$1.790+044.841+03$

$1.810+044,698+03$

$1.871+044.297+03$

$1.871+044.297+03$

$1.910+044.049+03$

$1.930+043.938+03$

$1.986+043.643+03$

$2.003+043.562+03$
2 pts ref $=97 \mathrm{Ke} 01$

84 pts ref $=001 \mathrm{Ch} 01$

$9.003+033.179+04$

$9.043+033.016+04$

$9.083+033.284+04$

$9.123+033.096+04$

$9.283+032.867+04$

$9.634+032.585+04$

$1.044+042.068+04$

$1.125+041.692+04$

$1.205+041.406+04$

$1.306+041.133+04$

$1.386+049.639+03$

$1.467+048.289+03$

$1.547+047.181+03$

$1.568+046.933+03$

$1.628+046.254+03$

$1.709+045.483+03$

$1.770+045.002+03$

$1.830+044.554+03$

$1.871+044.293+03$

$1.949+043.830+03$

$2.003+043.561+03$

$\mathrm{Z}=29, \mathrm{Cu}$

$\mathrm{eV}$, barns per atom pairs

6 pts ref $=001 \mathrm{An} 01$

$2.210+042.570+03 \quad 2.499+041.830+03 \quad 3.206+049.200+02 \quad 3.665+047.100+02$ $4.423+043.730+02 \quad 5.065+042.500+02$

$\mathrm{Z}=29, \mathrm{Cu}$

$\mathrm{eV}$, barns per atom pairs

47 pts ref $=78 \mathrm{Gi0} 1$

$9.620+063.269+00$

$9.870+063.282+00 \quad 1.013+073.289+00$

$1.037+073.291+00$

$1.061+073.297+00$

$1.086+073.305+00$

$1.112+073.315+00$

$1.134+073.322+00$

$1.150+073.326+00$

$1.417+073.446+00$

$1.440+073.458+00$

$1.464+073.454+00$

$1.486+073.453+00$

$1.514+073.495+00$

$1.537+073.498+00$

$1.560+073.530+00$

$1.587+073.537+00$

$1.610+073.553+00$

$1.635+073.570+00$

$1.664+073.587+00$

$1.687+073.605+00$

$1.711+073.611+00$

$1.736+073.611+00$

$1.761+073.634+00$

$1.788+073.622+00$

$1.814+073.618+00$

$1.839+073.643+00$

$1.864+073.659+00$ 
$\mathrm{Z}=40, \mathrm{Zr}$

$1.722+042.448+03$

$1.786+041.527+03$

$1.825+043.946+03$

$\mathrm{Z}=41, \mathrm{Nb}$

$1.337+045.500+03$

$2.210+049.400+03$

$4.423+041.420+03$

$\mathrm{Z}=41, \mathrm{Nb}$

$1.857+042.055+03$

$1.896+046.117+03$

$2.011+041.202+04$

$\mathrm{Z}=41, \mathrm{Nb}$

$4.300+041.549+03$

$\mathrm{Z}=42, \mathrm{Mo}$

$6.400+034.591+04$

$\mathrm{Z}=42$, Mo

$1.337+046.200+03$

$\mathrm{Z}=42$, Mo

$1.966+041.333+03$

$2.011+045.297+03$

$\mathrm{Z}=42$, Mo

$5.950+046.872+02$

$Z=42, M_{0}$

$5.110+051.403+0$

$1.330+068.001+00$

$\mathrm{Z}=45, \mathrm{Rh}$

$1.337+049.100+03$

$2.210+042.100+03$

$5.065+041.470+03$

$\mathrm{Z}=45, \mathrm{Rh}$

$\mathrm{eV}$, barns per atom pairs

5 pts $\quad$ ref $=003 \mathrm{Te} 01$

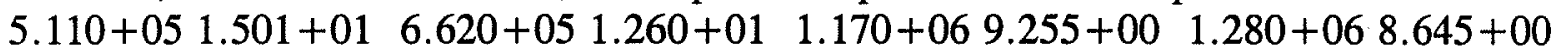
$1.330+068.488+00$

12 pts ref $=002 \mathrm{Ta} 01$

$1.748+042.081+03 \quad 1.767+04 \quad 1.913+03 \quad 1.780+041.447+03$

$1.799+041.643+03 \quad 1.806+041.846+03 \quad 1.818+042.243+03$

$1.838+041.247+04 \quad 1.876+04 \quad 1.260+04 \quad 1.934+041.272+04$

$\mathrm{eV}$, barns per atom pairs

10 pts ref $=001 \mathrm{An} 01$

$1.497+04 \quad 4.200+03 \quad 1.744+042.650+03 \quad 1.963+04 \quad 1.130+04$

$2.499+046.810+03 \quad 3.206+043.400+03 \quad 3.665+042.390+03$

$5.065+049.800+02$

$\mathrm{eV}$, barns per atom pairs

9 pts ref $=002 \mathrm{Ta} 01$

$1.870+04 \quad 1.884+03 \quad 1.876+042.093+03 \quad 1.883+042.329+03$

$1.902+041.247+04 \quad 1.928+04 \quad 1.569+04 \quad 1.966+041.208+04$

$\mathrm{eV}$, barns per atom pairs

2 pts ref $=97 \mathrm{Ro} 01$

$5.950+047.337+02$

2 pts ref $=97 \mathrm{Ke} 01$

$8.041+032.524+04$

3 pts ref $=001 \mathrm{An} 01$

$1.497+043.900+03 \quad 1.744+042.930+03$

8 pts ref $=002 \mathrm{Ta} 01$

$\begin{array}{llllll}1.985+04 & 1.550+03 & 1.998+04 & 1.585+03 & 2.005+04 & 3.298+03\end{array}$

$2.018+049.415+03 \quad 2.030+041.184+04 \quad 2.056+04 \quad 1,255+04$

$\mathrm{eV}$, barns per atom pairs

$1 \mathrm{pt} \quad$ ref $=97 \mathrm{Ro} 01$

$\mathrm{eV}$, barns per atom pairs

5 pts ref $=003 \mathrm{Te} 01$

$6.620+051.168+01 \quad 1.170+068.850+00 \quad 1.280+068.122+00$

$\mathrm{eV}$, barns per atom pairs

9 pts ref $=001 \mathrm{An} 01$

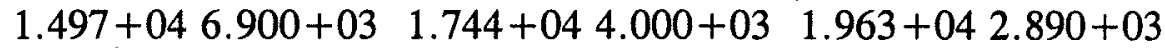

$3.206+044.750+03 \quad 3.665+043.300+03 \quad 4.423+042.020+03$ (1) 
$\mathrm{Z}=50, \mathrm{Sn}$

$9.620+067.641+00$

$1.061+077.822+00$

$1.158+078.028+00$

$1.261+078.210+00$

$1.361+078.438+00$

$1.464+078.662+00$

$1.560+078.860+00$

$1.664+078.974+00$

$1.761+079.078+00$

$1.864+079.156+00$

$1.962+079.248+00$

$2.057+079.398+00$

$2.157+079.484+00$

$2.264+079.600+07$

$2.365+079.668+00$

$2.464+079.787+00$

$2.556+079.880+00$

$2.656+079.982+00$

$2.757+071.006+01$

$2.856+071.012+01$

$2.958+071.033+01$

$3.068+071.037+01$

$3.161+071.035+01$

$3.262+071.046+01$

$3.360+071.060+01$

$3.466+071.067+01$

$3.573+071.068+01$

$3.679+071.084+01$

$4.939+071.160+01$

$5.136+071.161+01$

$5.340+071.175+01$

$1.025+081.347+01$
$\mathrm{eV}$, barns per atom pairs

$9.870+067.686+00 \quad 1.013+077.740+00$

$1.087+077.870+00 \quad 1.112+077.902+00$

$1.186+078.060+00$

$1.286+078.279+00$

$1.210+078.091+00$

$1.311+078.325+00$

$1.387+078.486+00$

$1.411+078.555+00$

$1.487+078.757+00$

$1.585+078.913+00$

$1.688+079.013+00$

$1.512+078.785+00$

$1.611+078.896+00$

$1.710+079.043+00$

$1.790+079.098+00$

$1.814+079.091+00$

$1.888+079.179+00$

$1.988+079.275+00$

$2.086+079.392+00$

$2.180+079.506+00$

$2.279+079.595+00$

$2.383+079.700+00$

$2.490+079.842+00$

$2.583+079.882+00$

$2.683+071.003+01$

$2.783+071.013+01$

$2.884+071.017+01$

$2.985+071.031+01$

$3.085+071.036+01$

$3.186+071.046+01$

$3.278+071.061+01$

$3.377+071.056+01$

$3.479+071.064+01$

$3.582+071.072+01$

$4.790+071.150+01$

$4.987+071.165+01$

$5.185+071.173+01$

$5.388+071.175+01$

$1.912+079.229+00$

$2.015+079.290+00$

$2.114+079.421+00$

$2.207+079.508+00$

$2.308+079.654+00$

$2.405+079.704+00$

$2.515+079.876+00$

$2.612+079.965+00$

$2.710+071.000+01$

$2.811+071.013+01$

$2.913+071.018+01$

$3.015+071.033+01$

$3.112+071.032+01$

$3.211+071.042+01$

$3.308+071.050+01$

$3.410+071.064+01$

$3.512+071.060+01$

$3.615+071.081+01$

$4.840+071.165+01$

$5.037+071.163+01$

$5.238+071.168+01$

$1.004+081.335+01$

$1.035+081.351+01$

$1.046+081.335+01$
8 pts ref $=78 \mathrm{Gi0} 1$

$1.037+077.776+00$

$1.136+077.973+00$

$1.234+078.168+00$

$1.336+078.402+00$

$1.439+078.617+00$

$1.537+078.800+00$

$1.635+078.928+00$

$1.735+079.078+00$

$1.839+079.144+00$

$1.936+079.219+00$

$2.037+079.361+00$

$2.136+075.418+00$

$2.236+079.537+00$

$2.337+079.625+00$

$2.433+079.736+00$

$2.528+079.825+00$

$2.630+079.978+00$

$2.737+071.004+01$

$2.839+071.014+01$

$2.942+071.024+01$

$3.045+071.028+01$

$3.139+071.039+01$

$3.238+071.052+01$

$3.339+071.056+01$

$3.443+071.059+01$

$3.545+071.067+01$

$3.647+071.092+01$

$4.889+071.160+01$

$5.087+071.168+01$

$5.286+071.173+01$

$1.013+081.343+01$

$1.056+081.350+01$
$\mathrm{Z}=54, \mathrm{Xe}$

$8.600+011.960+07$

$1.050+022.710+07$

$1.350+025.360+06$

$1.417+023.970+06$

$1.443+022.880+06$

$1.500+022.180+06$

$1.900+029.010+05$

$2.157+021.200+06$

$2.500+021.470+06$
$\mathrm{eV}$, barns per atom pairs

$9.000+012.420+07$

$1.100+022.380+07$

$1.400+023.570+06$

$1.424+023.370+06$

$1.450+022.850+06$

$1.600+021.360+06$

$2.000+029.730+05$

$2.200+021.220+06$

$2.600+021.530+06$
$9.500+012.760+07$

$1.200+021.500+07$

$1.410+023.400+06$

$1.433+022.830+06$

$1.460+022.630+06$

$1.700+029.890+05$

$2.099+021.100+06$

$2.300+021.310+06$

$2.700+021.570+06$
10 pts $\quad$ ref $=003 \mathrm{Su} 01$

$1.000+022.870+07$

$1.300+027.830+06$

$1.414+023.600+06$

$1.439+023.150+06$

$1.470+022.540+06$

$1.800+028.790+05$

$2.110+021.150+06$

$2.400+021.390+06$

$2.800+021.590+06$ 
$\mathrm{Z}=60, \mathrm{Nd}$

$\mathrm{eV}$, barns per atom pairs

$12 \mathrm{pts}$ ref $=002 \mathrm{Ma} 02$

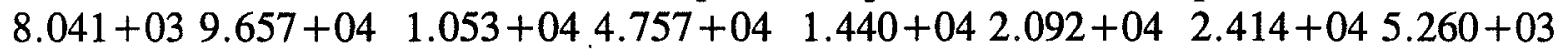

$3.080+042.740+03 \quad 3.500+041.960+03 \quad 5.201+043.560+03 \quad 6.430+042.050+03$

$7.083+041.600+03$

$7.287+041.480+03$

$8.100+041.120+03$

$8.430+041.000+03$

$\mathrm{Z}=62, \mathrm{Sm}$

$\mathrm{eV}$, barns per atom pairs

11 pts ref $=002 \mathrm{Ma} 02$

$1.053+045.391+04 \quad 1.440+042.374+04 \quad 2.414+04 \quad 6.010+03 \quad 3.080+043.160+03$

$\begin{array}{lllllll}3.500+042.220+03 & 5.201+04 & 3.990+03 & 6.430+04 & 2.250+03 & 7.083+04 & 1.800+03\end{array}$

$7.287+041.660+03$

$8.100+041.260+03$

$8.430+041.130+03$

$\mathrm{Z}=63, \mathrm{Eu}$

$5.954+042.872+03$

$\mathrm{Z}=64, \mathrm{Gd}$ $\mathrm{eV}$, barns per atom pairs

$1 \mathrm{pt} \quad \mathrm{ref}=99 \mathrm{Ka} 01$

$1.053+046.059+04$

$\mathrm{eV}$, barns per atom pairs

11 pts ref $=002 \mathrm{Ma} 02$

$3.500+042.550+03$

$1.440+042.690+042.414+046.920+03 \quad 3.080+043.600+03$

$7.287+041.860+03$

$5.201+044.550+03$

$6.430+042.570+03 \quad 7.083+042.000+03$

$\mathrm{Z}=64, \mathrm{Gd}$

$5.950+043.462+03$

$\mathrm{eV}$, barns per atom pairs

$1 \mathrm{pt} \quad \mathrm{ref}=97 \mathrm{Ro} 01$

$\mathrm{Z}=64, \mathrm{Gd}$

$\mathrm{eV}$, barns per atom pairs

$1 \mathrm{pt} \quad \mathrm{ref}=99 \mathrm{Ka} 01$

$5.954+043.050+03$

$\mathrm{Z}=64, \mathrm{Gd}$

$5.960+043.071+03$

$\mathrm{Z}=65, \mathrm{~Tb}$

$5.954+043.178+03$

$\mathrm{eV}$, barns per atom pairs

$1 \mathrm{pt} \quad$ ref $=96 \mathrm{Er} 01$

$\mathrm{Z}=65, \mathrm{~Tb}$

$5.960+043.086+03$

$\mathrm{eV}$, barns per atom pairs

$1 \mathrm{pt} \quad \mathrm{ref}=99 \mathrm{Ka} 01$

$\mathrm{Z}=66, \mathrm{Dy}$

$\mathrm{eV}$, barns per atom pairs

$11 \mathrm{pts}$ ref $=002 \mathrm{Ma} 02$

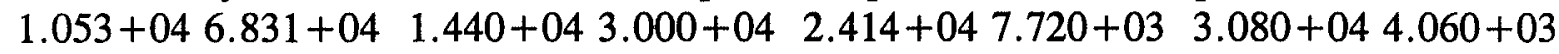

$3.500+042.900+03 \quad 5.201+041.040+03 \quad 6.430+042.840+03 \quad 7.083+042.220+03$

$7.287+042.100+03$

$8.100+041.570+03 \quad 8.430+041.410+03$

$\mathrm{Z}=66, \mathrm{Dy}$

$4.300+041.497+03$

$\mathrm{Z}=66$, Dy

$5.954+043.396+03$

$\mathrm{eV}$, barns per atom pairs

$1 \mathrm{pt} \quad \mathrm{ref}=97 \mathrm{Ro} 01$

$\mathrm{eV}$, barns per atom pairs

$1 \mathrm{pt} \quad \mathrm{ref}=99 \mathrm{Ka} 01$ 
$1.791+037.174+05$ $1.801+037.388+05$ $1.811+038.210+05$ $1.821+039.061+05$ $1.831+039.927+05$ $1.840+039.966+05$ $1.851+03 \quad 1.005+06$ $1.861+031.017+06$ $1.871+031.032+06$ $1.881+031.044+06$ $1.891+031.037+06$ $1.901+031.033+06$ $1.911+031.026+06$ $1.921+031.023+06$ $1.931+031.021+06$ $1.941+031.023+06$ $1.951+031.007+06$ $1.961+039.986+05$ $1.971+039.940+05$ $1.981+039.870+05$ $1.991+039.843+05$ $2.001+039.841+05$ $2.011+039.666+05$ $2.021+039.578+05$ $2.031+039.461+05$ $2.041+039.378+05$ $2.051+039.315+05$ $2.061+039.128+05$ $2.071+039.154+05$ $2.081+039.022+05$ $2.091+038.943+05$ $2.101+038.861+05$ $2.111+038.843+05$ $2.121+038.753+05$ $2.131+038.669+05$ $2.141+038.611+05$ $2.151+038.466+05$ $2.161+038.503+05$ $2.171+038.440+05$ $2.181+038.616+05$ $2.191+039.999+05$ $2.201+039.755+05$ $2.211+039.347+05$ $2.221+039.164+05$
$1.793+037.197+05$ $1.803+037.686+05$ $1.813+038.342+05$ $1.823+039.307+05$ $1.833+039.943+05$ $1.843+039.980+05$ $1.853+031.002+06$ $1.863+031.021+06$ $1.873+031.039+06$ $1.883+031.040+06$ $1.893+031.031+06$ $1.903+031.031+06$ $1.913+03 \quad 1.020+06$ $1.923+031.026+06$ $1.933+031.023+06$ $1.943+03 \quad 1.018+06$ $1.953+039.938+05$ $1.963+039.948+05$ $1.973+039.846+05$ $1.983+039.835+05$ $1.993+039.772+05$ $2.003+039.750+05$ $2.013+039.658+05$ $2.023+039.548+05$ $2.033+039.449+05$ $2.043+039.337+05$ $2.053+039.291+05$ $2.063+039.157+05$ $2.073+039.039+05$ $2.083+039.024+05$ $2.093+038.916+05$ $2.103+038.872+05$ $2.113+038.791+05$ $2.123+038.767+05$ $2.133+038.666+05$ $2.143+038.592+05$ $2.153+038.541+05$ $2.163+038.460+05$ $2.173+038.459+05$ $2.183+038.695+05$ $2.193+031.030+06$ $2.203+039.604+05$ $2.213+039.252+05$ $2.223+039.156+05$
$1.796+037.241+05$ $1.806+037.887+05$ $1.816+038.579+05$ $1.826+039.647+05$ $1.836+039.949+05$ $1.846+039.935+05$ $1.856+031.015+06$ $1.866+03 \quad 1.019+06$ $1.876+031.039+06$ $1.886+031.042+06$ $1.896+031.033+06$ $1.906+031.034+06$ $1.916+031.017+06$ $1.926+031.024+06$ $1.936+031.022+06$ $1.946+031.013+06$ $1.956+031.005+06$ $1.966+039.953+05$ $1.976+039.793+05$ $1.986+039.876+05$ $1.996+039.821+05$ $2.006+039.675+05$ $2.016+039.551+05$ $2.026+039.521+05$ $2.036+039.381+05$ $2.046+039.325+05$ $2.056+039.236+05$ $2.066+039.141+05$ $2.076+039.080+05$ $2.086+038.938+05$ $2.096+038.895+05$ $2.106+038.835+05$ $2.116+038.776+05$ $2.126+038.675+05$ $2.136+038.692+05$ $2.146+038.578+05$ $2.156+038.521+05$ $2.166+038.510+05$ $2.176+038.480+05$ $2.186+039.035+05$ $2.196+031.026+06$ $2.206+039.485+05$ $2.216+039.274+05$ $2.226+039.057+05$
$1.798+037.311+05$ $1.808+038.041+05$ $1.818+038.777+05$ $1.828+039.816+05$ $1.838+031.003+06$ $1.848+039.961+05$ $1.858+031.014+06$ $1.868+031.032+06$ $1.878+031.038+06$ $1.888+031.036+06$ $1.898+031.041+06$ $1.908+031.020+06$ $1.918+031.025+06$ $1.928+03 \quad 1.024+06$ $1.938+031.020+06$ $1.948+03 \quad 1.016+06$ $1.958+031.001+06$ $1.968+039.879+05$ $1.978+039.891+05$ $1.988+039.813+05$ $1.998+039.790+05$ $2.008+039.637+05$ $2.018+039.593+05$ $2.028+039.508+05$ $2.038+039.378+05$ $2.048+039.369+05$ $2.058+039.241+05$ $2.068+039.153+05$ $2.078+039.042+05$ $2.088+039.002+05$ $2.098+038.913+05$ $2.108+038.793+05$ $2.118+038.730+05$ $2.128+038.683+05$ $2.138+038.595+05$ $2.148+038.630+05$ $2.158+038.502+05$ $2.168+038.482+05$ $2.178+038.541+05$ $2.188+039.405+05$ $2.198+031.002+06$ $2.208+039.422+05$ $2.218+039.181+05$ $2.228+039.062+05$ 
$7.483+072.482+01$ $7.747+072.519+01$ $8.109+072.520+01$ $9.654+072.613+01$ $1.005+082.622+01$ $1.045+082.627+01$ $1.085+082.643+01$ $1.164+082.705+01$ $1.206+082.724+01$ $1.245+082.727+01$ $1.301+082.708+01$ $1.480+082.797+01$ $1.560+082.815+01$

$\mathrm{Z}=74, \mathrm{~W}$

$1.453+035.889+05$ $1.493+035.539+05$

$1.533+035.213+05$

$1.573+034.810+05$ $1.613+034.605+05$ $1.653+034.483+05$ $1.693+034.008+05$ $1.733+033.745+05$ $1.761+033.510+05$ $1.781+033.431+05$

$1.801+033.420+05$

$1.806+033.644+05$

$1.810+033.682+05$

$1.814+033.689+05$

$1.818+033.973+05$

$1.822+034.371+05$

$1.826+034.859+05$

$1.830+035.212+05$

$1.834+035.719+05$

$1.838+036.058+05$

$1.842+036.746+05$

$1.846+037.428+05$

$1.850+037.466+05$

$1.854+037.586+05$

$1.858+038.004+05$

$1.862+038.024+05$

$1.866+037.903+05$

$1.870+037.852+05$

$1.874+038.030+05$
$7.525+072.501+01$

$7.850+072.513+01$

$9.356+072.597+01$

$9.753+072.619+01$

$1.015+082.624+01$

$1.055+082.636+01$

$1.131+082.677+01$

$1.174+082.715+01$

$1.213+082.725+01$

$1.256+082.740+01$

$1.426+082.765+01$

$1.499+082.806+01$

$1.579+082.818+01$
$7.555+072.496+01$

$7.949+072.519+01$

$9.456+072.617+07$

$9.851+072.631+01$

$1.025+082.618+01$

$1.065+082.630+01$

$1.142+082.665+01$

$1.185+082.706+01$

$1.222+082.726+01$

$1.261+082.742+01$

$1.440+082.779+01$

$1.520+082.808+01$

$1.598+082.816+01$
$7.652+072.504+01$

$8.028+072.528+01$

$9.555+072.603+01$

$9.952+072.615+01$

$1.034+082.635+01$

$1.075+082.675+01$

$1.153+082.719+01$

$1.196+082.712+01$

$1.234+082.729+01$

$1.283+082.754+01$

$1.460+082.778+01$

$1.541+082.798+01$

$1.618+082.830+01$
$\mathrm{eV}$, barns per atom pairs

$1.463+035.810+05$

$1.503+035.503+05$

$1.543+035.084+05$

$1.583+034.892+05$

$1.623+034.507+05$

$1.663+034.234+05$

$1.703+033.981+05$

$1.743+033.657+05$

$1.766+033.467+05$

$1.786+033.431+05$

$1.803+033.709+05$

$1.807+033.601+05$

$1.811+033.771+05$

$1.815+033.789+05$

$1.819+034.103+05$

$1.823+034.668+05$

$1.827+034.937+05$

$1.831+035.381+05$

$1.835+035.846+05$

$1.839+036.200+05$

$1.843+036.967+05$

$1.847+037.468+05$

$1.851+037.466+05$

$1.855+037.747+05$

$1.859+038.066+05$

$1.863+037.957+05$

$1.867+037.867+05$

$1.871+037.891+05$

$1.875+037.966+05$
$1.473+035.753+05$

$1.513+035.361+05$

$1.553+034.994+05$

$1.593+034.740+05$

$1.633+034.478+05$

$1.673+034.189+05$

$1.713+033.930+05$

$1.753+033.660+05$

$1.771+033.373+05$

$1.791+033.326+05$

$1.804+033.652+05$

$1.808+033.682+05$

$1.812+033.615+05$

$1.816+033.815+05$

$1.820+034.270+05$

$1.824+034.716+05$

$1.828+035.088+05$

$1.832+035.435+05$

$1.836+035.911+05$

$1.840+036.280+05$

$1.844+037.198+05$

$1.848+037.551+05$

$1.852+037.432+05$

$1.856+037.862+05$

$1.860+038.027+05$

$1.864+038.053+05$

$1.868+037.915+05$

$1.872+037.877+05$

$1.876+038.050+05$ pts $\quad$ ref $=003 L e 01$

$1.483+035.611+05$

$1.523+035.310+05$

$1.563+035.022+05$

$1.603+034.681+05$

$1.643+034.331+05$

$1.683+034.059+05$

$1.723+033.816+05$

$1.756+033.394+05$

$1.776+033.369+05$

$1.796+033.314+05$

$1.805+033.671+05$

$1.809+033.659+05$

$1.813+033.728+05$

$1.817+033.843+05$

$1.821+034.363+05$

$1.825+034.789+05$

$1.829+035.192+05$

$1.833+035.554+05$

$1.837+035.984+05$

$1.841+036.344+05$

$1.845+037.423+05$

$1.849+037.462+05$

$1.853+037.445+05$

$1.857+037.887+05$

$1.861+038.097+05$

$1.865+037.880+05$

$1.869+037.742+05$

$1.873+038.009+05$

$1.877+038.013+05$ 
$2.046+031.103+06$ $2.049+031.089+06$ $2.052+031.094+06$ $2.056+031.079+06$ $2.059+031.078+06$ $2.062+031.063+06$ $2.066+031.062+06$ $2.069+031.044+06$ $2.072+031.043+06$ $2.076+03 \quad 1.030+06$ $2.079+031.068+06$ $2.082+031.084+06$ $2.086+031.067+06$ $2.089+031.079+06$ $2.092+031.070+06$ $2.096+031.070+06$ $2.099+031.076+06$

$2.102+031.063+06$

$2.106+03 \quad 1.059+06$

$2.109+031.039+06$

$2.112+031.030+06$

$2.116+031.037+06$

$2.119+031.023+06$

$2.122+031.018+06$

$2.126+031.024+06$

$2.129+031.011+06$

$2.132+031.004+06$

$2.136+031.006+06$ $2.139+039.826+05$ $2.142+039.911+05$

$2.146+031.003+06$

$2.149+039.887+05$ $2.152+039.924+05$ $2.161+031.013+06$ $2.171+031.004+06$ $2.181+039.964+05$ $2.191+039.759+05$ $2.201+039.708+05$ $2.211+039.574+05$ $2.221+039.580+05$ $2.231+039.498+05$ $2.241+039.417+05$ $2.251+039.322+05$ $2.261+039.378+05$
$2.046+031.091+06$ $2.050+031.092+06$ $2.053+031.084+06$ $2.056+031.070+06$ $2.060+031.071+06$ $2.063+031.060+06$ $2.066+031.060+06$ $2.070+031.042+06$ $2.073+031.046+06$ $2.076+031.058+06$ $2.080+031.072+06$ $2.083+031.082+06$ $2.086+031.076+06$ $2.090+031.079+06$ $2.093+031.072+06$ $2.096+031.069+06$ $2.100+031.066+06$ $2.103+031.070+06$ $2.106+031.060+06$ $2.110+031.039+06$ $2.113+031.040+06$ $2.116+031.026+06$ $2.120+031.017+06$ $2.123+031.020+06$ $2.126+031.012+06$ $2.130+031.008+06$ $2.133+031.002+06$ $2.136+039.951+05$ $2.140+039.837+05$ $2.143+031.000+06$ $2.146+039.831+05$ $2.150+039.936+05$ $2.153+031.003+06$ $2.163+031.014+06$ $2.173+031.001+06$ $2.183+039.892+05$ $2.193+039.710+05$ $2.203+039.585+05$ $2.213+039.512+05$ $2.223+039.487+05$ $2.233+039.387+05$ $2.243+039.439+05$ $2.253+039.429+05$ $2.263+039.394+05$
$2.047+03 \quad 1.090+06 \quad 2.048+03 \quad 1.100+06$ $2.051+031.092+062.051+031.085+06$ $2.054+031.085+06 \quad 2.055+031.078+06$ $2.057+031.080+062.058+031.079+06$ $2.061+031.069+06 \quad 2.061+03 \quad 1.072+06$ $2.064+031.061+06 \quad 2.065+031.062+06$ $2.067+031.053+06 \quad 2.068+031.051+06$ $2.071+031.045+062.071+031.043+06$ $2.074+031.047+062.075+031.060+06$ $2.077+031.057+06 \quad 2.078+031.053+06$ $2.081+031.056+06 \quad 2.081+031.080+06$ $2.084+031.081+06 \quad 2.085+031.083+06$ $2.087+031.082+062.088+031.079+06$ $2.091+031.077+062.091+031.080+06$ $2.094+031.064+06 \quad 2.095+031.068+06$ $2.097+031.067+062.098+031.069+96$ $2.101+031.066+06 \quad 2.101+03 \quad 1.076+06$ $2.104+031.064+06 \quad 2.105+031.053+06$ $2.107+03 \quad 1.062+06 \quad 2.108+03 \quad 1.045+06$ $2.111+031.050+06 \quad 2.111+03 \quad 1.039+06$ $2.114+031.032+06 \quad 2.115+031.020+06$ $2.117+031.029+06 \quad 2.118+031.032+06$ $2.121+031.030+06 \quad 2.121+031.017+06$ $2.124+031.016+06 \quad 2.125+031.005+06$ $2.127+031.011+06 \quad 2.128+03 \quad 1.012+06$ $2.131+031.020+062.131+031.006+06$ $2.134+039.975+05 \quad 2.135+031.002+06$ $2.137+031.003+062.138+039.990+05$ $2.141+031.009+062.141+039.894+05$ $2.144+039.878+052.145+039.968+05$ $2.147+039.913+05 \quad 2.148+039.991+05$ $2.151+031.003+062.151+039.866+05$ $2.156+031.010+062.158+031.006+06$ $2.166+03 \quad 1.008+06 \quad 2.168+03 \quad 1.012+06$ $2.176+03 \quad 1.005+06 \quad 2.178+03 \quad 1.007+06$ $2.186+039.787+052.188+039.785+05$ $2.196+039.609+052.198+039.582+05$ $2.206+039.602+052.208+039.521+05$ $2.216+039.614+052.218+039.568+05$ $2.226+039.402+052.228+039.509+05$ $2.236+039.488+05 \quad 2.238+039.441+05$ $2.246+039.459+05 \quad 2.248+039.389+05$ $2.256+039.349+05 \quad 2.258+059.367+05$ $2.266+039.408+052.268+039.484+05$ 
$2.364+072.257+01$ $2.463+072.295+01$ $2.562+072.316+01$ $2.657+072.340+01$ $2.759+072.374+01$ $2.861+072.402+01$ $2.961+072.424+01$ $3.177+072.472+01$ $3.374+072.519+01$ $3.577+072.547+01$ $3.772+072.588+01$ $3.975+072.623+01$ $4.176+072.659+01$ $4.378+072.699+01$ $4.584+072.724+01$ $4.781+072.744+01$ $4.981+072.764+01$ $5.183+072.810+01$ $5.386+072.843+01$ $5.574+072.857+01$ $5.757+072.873+01$ $5.970+072.903+01$ $6.171+072.919+01$ $6.381+072.955+01$ $6.573+072.967+01$ $6.779+072.999+01$ $6.980+072.993+01$ $7.174+073.013+01$ $7.374+073.049+01$ $7.663+073.051+01$ $8.050+073.098+01$ $8.451+073.117+01$ $8.848+073.145+01$ $9.245+073.177+01$ $9.649+073.187+01$ $1.004+083.215+01$ $1.044+083.245+01$ $1.086+083.260+01$ $1.128+083.293+01$ $1.163+083.287+01$ $1.204+083.316+01$ $1.246+083.338+01$ $1.319+083.362+01$ $1.399+083.397+01$
$2.390+072.269+01$ $2.485+072.294+01$ $2.580+072.322+01$ $2.682+072.342+01$ $2.786+072.369+01$ $2.888+072.406+01$ $2.989+072.425+01$ $3.227+012.483+01$ $3.426+072.518+01$ $3.627+072.570+01$ $3.823+072.592+01$ $4.021+072.630+01$ $4.223+072.661+01$ $4.424+072.691+01$ $4.636+072.721+01$ $4.829+072.756+01$ $5.033+072.776+01$ $5.235+072.807+01$ $5.440+072.845+01$ $5.616+072.854+01$ $5.813+072.869+01$ $6.026+072.901+01$ $6.227+072.932+01$ $6.427+072.949+01$ $6.620+072.964+01$ $6.818+072.981+01$ $7.020+073.003+01$ $7.215+073.036+01$ $7.422+073.049+01$ $7.765+073.081+01$ $8.154+073.082+01$ $8.556+073.137+01$ $8.947+073.148+01$ $9.348+073.188+01$ $9.751+073.198+01$ $1.014+083.227+01$ $1.055+083.254+01$ $1.097+083.255+01$ $1.136+083.299+01$ $1.174+083.305+01$ $1.215+083.318+01$ $1.260+083.333+01$ $1.340+083.384+01$ $1.421+083.404+01$
$2.413+072.274+01$ $2.513+072.301+01$ $2.608+072.335+01$ $2.708+072.360+01$ $2.810+072.380+01$ $2.910+072.403+01$ $3.080+072.448+01$ $3.277+072.491+01$ $3.478+072.535+01$ $3.672+012.573+01$ $3.873+072.606+01$ $4.070+072.639+01$ $4.276+072.681+01$ $4.482+072.704+01$ $4.683+072.740+01$ $4.877+072.761+01$ $5.081+072.799+01$ $5.282+072.825+01$ $5.485+072.847+01$ $5.665+072.870+01$ $5.865+072.900+01$ $6.067+072.923+01$ $6.279+072.935+01$ $6.467+072.969+01$ $6.674+072.979+01$ $6.876+073.011+01$ $7.079+072.999+01$ $7.279+073.040+01$ $7.479+073.050+01$ $7.854+073.064+01$ $8.257+073.108+01$ $8.656+073.135+01$ $9.051+073.151+01$ $9.454+073.178+01$ $9.842+073.203+01$ $1.024+083.233+01$ $1.065+083.269+01$ $1.107+083.287+01$ $1.145+083.294+01$ $1.183+083.300+01$ $1.225+083.351+01$ $1.280+083.347+01$ $1.361+083.372+01$ $1.441+083.415+01$
$2.438+072.282+01$ $2.536+072.310+01$ $2.631+072.347+01$ $2.735+072.366+01$ $2.837+072.390+01$ $2.937+072.419+01$ $3.126+072.458+01$ $3.324+072.504+01$ $3.528+072.538+01$ $3.721+072.577+01$ $3.923+072.617+01$ $4.119+072.657+01$ $4.326+072.682+01$ $4.533+072.716+01$ $4.730+072.742+01$ $4.933+072.774+01$ $5.136+072.809+01$ $5.338+072.827+01$ $5.526+072.865+01$ $5.706+072.869+01$ $5.923+072.896+01$ $6.121+072.922+01$ $6.328+072.940+01$ $6.523+072.946+01$ $6.726+072.974+01$ $6.930+073.011+01$ $7.127+073.015+01$ $7.335+073.031+01$ $7.551+073.042+01$ $7.949+073.084+01$ $8.353+073.111+01$ $8.749+073.124+01$ $9.150+073.163+01$ $9.551+073.181+01$ $9.940+073.206+01$ $1.035+083.229+01$ $1.075+083.248+01$ $1.117+083.280+01$ $1.155+083.302+01$ $1.192+083.310+01$ $1.234+083.324+01$ $1.302+083.360+01$ $1.379+083.385+01$ $1.458+083.422+01$ 


\begin{tabular}{llc}
$57 \mathrm{La}$ & 1 & 13 \\
$58 \mathrm{Ce}$ & 2 & 13 \\
$59 \mathrm{Pr}$ & 2 & 13 \\
$60 \mathrm{Nd}$ & 2 & 13 \\
$62 \mathrm{Sm}$ & 1 & 11 \\
$63 \mathrm{Eu}$ & 1 & 1 \\
$64 \mathrm{Gd}$ & 4 & 14 \\
$65 \mathrm{~Tb}$ & 2 & 2 \\
$66 \mathrm{Dy}$ & 4 & 14 \\
$67 \mathrm{Ho}$ & 2 & 12 \\
$68 \mathrm{Er}$ & 4 & 14 \\
$70 \mathrm{Yb}$ & 1 & 2 \\
$73 \mathrm{Ta}$ & 5 & 426 \\
$74 \mathrm{~W}$ & 2 & 507 \\
$78 \mathrm{Pt}$ & 1 & 2 \\
$79 \mathrm{Au}$ & 3 & 7 \\
$82 \mathrm{~Pb}$ & 2 & 244 \\
$92 \mathrm{U}$ & 2 & 3 \\
\hline
\end{tabular}

48 Elements Total Data Points Extracted: 3357 